\title{
On the linear prediction problem for certain stochastic processes
}

\author{
By Harald Cramér
}

1. Consider an infinite sequence of complex-valued random variables

$$
\ldots, x_{-2}, x_{-1}, x_{0}, x_{1}, x_{2}, \ldots
$$

with finite second order mean values. For the sake of simplicity, we shall assume throughout that all first order mean values of the $x_{n}$ reduce to zero, while there is at least one $x_{n}$ having a variance different from zero:

$$
\begin{gathered}
E x_{n}=0 \text { for all } n, \\
E\left|x_{n}\right|^{2}>0 \text { for some } n .
\end{gathered}
$$

The covariance function of the $x_{n}$ sequence is

$$
R(m, n)=E\left(x_{m} \overline{x_{n}}\right) \text {. }
$$

We may interpret $x_{n}$ as a measure of the state of some observed variable system at the time point $n t$, where $t$ is a given quantity. The sequence of the $x_{n}$, with $n=\cdots,-1,0,1, \ldots$, will then represent the temporal development of this system, and will constitute a stochastic process with discrete time. In the sequel, we shall always take $t=1$, so that the subscript $n$ may be directly regarded as measuring time.

The prediction problem for a process of this kind is the problem of predicting the state of the process at some future time point, when its past development is assumed to be more or less known. In this paper we shall only be concerned with linear least squares prediction. Thus we shall want to find the "best possible" prediction of a certain $x_{n}$ by means of linear operations acting on certain variables belonging to the past of the process, interpreting the "best possible" in the sense of minimizing the mean value of the squared error of prediction.

Consider the expression

$$
\underset{c_{0} \ldots \ldots c_{q}}{\operatorname{Min}} E\left|x_{n}-c_{0} x_{n-p}-c_{1} x_{n-p-1}-\cdots-c_{q} x_{n-p-q}\right|^{2}=s_{n p q}^{2} \geqq 0,
$$

where $n, p$ and $q$ are fixed integers with $p>0, q>0$, while the minimum has to be taken for all complex quantities $c_{0}, \ldots, c_{q}$. Then $s_{n p q}$ will be the least possible error of prediction, when $x_{n}$ has to be linearly predicted in terms of $x_{n-p}, x_{n-p-1}$, 
H. CRAMÉR, On the linear prediction problem for ceriain stochastic processes

$\ldots, x_{n-p-q}$. Obviously $s_{n p q}$ will never increase when $q$ increases, while $n$ and $p$ remain fixed. The limit

$$
\lim _{q \rightarrow \infty} s_{n p q}=\sigma_{n p} \geqq 0
$$

will thus always exist, and will be called the prediction error for $x_{n}$, when prediction is based on all the variables of the process up to and including $x_{n-p}$. We easily find that

$$
0 \leqq \sigma_{n 1} \leqq \sigma_{n 2} \leqq \cdots
$$

The same thing may be expressed a little differently, if we look at the question from the point of view of Hilbert space geometry. Consider the Hilbert space $H_{n-p}$ spanned by all the variables $x_{n-p}, x_{n-p-1}, \ldots$ The elements of this space are random variables, which are either finite linear combinations of $x_{n-p}, x_{n-p-1}, \ldots$, or limits in the mean of sequences of such combinations. The inner product and the norm are defined by the usual expressions

$$
(y, z)=E(y \bar{z}), \quad\|y\|=\left(E\left|y^{2}\right|\right)^{\frac{1}{2}} .
$$

By $P_{n-p}\left(x_{n}\right)$ we denote the projection of $x_{n}$ on $H_{n-p}$. Then $z=P_{n-p}\left(x_{n}\right)$ is the uniquely determined element of $H_{n-p}$ which minimizes the distance $\left\|x_{n}-z\right\|$. We shall call $P_{n-p}\left(x_{n}\right)$ the best possible linear prediction of $x_{n}$ in terms of $x_{n-p}, x_{n-p-1}, \ldots$ The corresponding error of prediction will be

$$
\sigma_{n p}=\left\|x_{n}-P_{n \rightarrow p}\left(x_{n}\right)\right\| \text {. }
$$

If $\sigma_{n p}=0$ for all $n$ and $p$, exact linear prediction is always possible. In this case every $x_{n}$ can be exactly represented in terms of variables belonging to an arbitrarily remote past of the process. A process of this kind will be called a deterministic process.

On the other hand, every process such that $\sigma_{n p}>0$ for at least one pair of values of $n$ and $p$, will be called non-deterministic. Every non-deterministic process may be represented as the sum of a deterministic component and a linear combination of certain innovations, which represent the "new" random impulses en. tering into the process at certain moments. In fact, it can be shown (cf. Cramér, 2) that for every non-deterministic process, there exists a uniquely determined, finite or infinite sequence of integers

such that

$$
\cdots<r_{-1}<r_{0}<r_{1}<\cdots
$$

$$
x_{n}=\sum_{r_{k} \leqq n} c_{n r_{k}} \xi_{r_{k}}+y_{n}
$$

Here $y_{n}$ is the deterministic component of the process, while the $\xi_{r_{k}}$ are random variables such that

$$
\begin{gathered}
E \xi_{r_{k}}=0, \quad E\left(\xi_{r j} \bar{\xi}_{r_{k}}\right)=\delta_{j k}, \\
E\left(x_{n} \bar{\xi}_{r_{k}}\right)=c_{n r_{k}}, \quad E\left(y_{n} \bar{\xi}_{r_{k}}\right)=0 .
\end{gathered}
$$

Further $\sum_{r_{k} \leqq n}\left|c_{n r_{k}}\right|^{2}$ is convergent, so that the series in the expression for $x_{n}$ con- 
verges in the mean. If $n$ is a member of the $r_{k}$ sequence, the quantity $c_{n n}$ is real and positive. Finally, the prediction error $\sigma_{n p}$ is given by the expression

$$
\sigma_{n p}^{2}=\sum_{n-p<r_{k} \leqq n}\left|c_{n r_{k}}\right|^{2} \text {. }
$$

It follows that $\sigma_{n 1}>0$ when and only when $n$ is a member of the $r_{k}$ sequence. Thus it will be seen that every $r_{k}$ is a point of indetermination, where the process receives an innovation proportional to $\xi_{r_{k}}$. On the other hand, when $n$ is different from all $r_{k}$, we have $\sigma_{n 1}=0$, so that $x_{n}$ can be exactly predicted in terms of the preceding variables $x_{n-1}, x_{n-2}, \ldots$, and there is no innovation corresponding to the time point $n$. Thus in the expression (1) of $x_{n}$, the non-deterministic component is a linear combination of the innovations received by the process in all its points of indetermination preceding or coinciding with the time point $n$.

2. The linear prediction problem has been thoroughly studied for the important class of stationary processes, which are characterized by the fact that the covariance function $R(m, n)=E\left(x_{m} \overline{x_{n}}\right)$ only depends on the time difference $m-n$. (We are not here concerned with the so called strictly stationary processes, which satisfy more stringent conditions.) For this class of processes, the sequence $r_{k}$ considered in the preceding section contains every integer $n$, and the representation (1) reduces to a decomposition theorem due to $H$. Wold, 1 . With respect to the general theory of stationary processes, and in particular the prediction problem, we may refer e.g. to the books by Wiener, 1 , and Doob, 1 .

One of the most important properties of the class of stationary processes is that they admit a spectral representation by means of a stochastic integral of Fourier type. Loève (1) has introduced a more general class of processes, which he calls harmonizable processes, and which possess spectral representations of a similar kind. In this paper, we shall consider the prediction problem for a group of harmonizable processes satisfying certain regularity conditions.

Consider a stochastic process such that $x_{n}$ is given by the stochastic integral

$$
x_{n}=\int_{0}^{2 \pi} e^{i n u} d z(u)
$$

where $z(u)$ denotes, for every $u$ in the interval $0 \leqq u \leqq 2 \pi$, a random variable such that

$$
E z(u)=0, \quad E(z(u) \overline{z(v)})=F(u, v),
$$

where $F(u, v)$-in general complex-valued-is of bounded variation over the square $C: 0 \leqq u, v \leqq 2 \pi$. Obviously

$$
F(v, u)=\overline{F(u, v)}
$$

while $F(u, u)$ is real and non-negative. Under these conditions, the stochastic integral (2) can be defined as a limit in the mean of certain Riemann sums, and the variable $x_{n}$ determines a harmonizable stochastic process. (Cf. also Cramér 1.)

In the sequel, we shall assume that the function $F(u, v)$ satisfies two addi. tional conditions, which we denote by $(A)$ and $(B)$. Thus we shall assume:

$$
F(u, v)=\int_{0}^{u} \int_{0}^{v} f(s, t) d s d t
$$




\section{H. CRAMÉR, On the linear prediction problem for certain stochastic processes}

where

(A) $f(s, t)$ belongs to $L^{2}$ over the square $C$.

(B) $f(s, t)$ is bounded in the vicinity of the diagonal $s=t$ of $C$, so that there exist positive constants $h$ and $M$ such that $|f(s, t)|<M$ for $|s-t|<h$.

The covariance function of the $x_{n}$ process is then given by

$$
\begin{aligned}
R(m, n)=E\left(x_{m} \overline{x_{n}}\right) & =\int_{0}^{2 \pi} \int_{0}^{2 \pi} e^{i(m u-\pi v)} d F(u, v) \\
& =\int_{0}^{2 \pi} \int_{0}^{2 \pi} e^{i(m u-n v)} f(u, v) d u d v .
\end{aligned}
$$

More generally, for any $g(u)$ and $h(u)$ belonging to $L^{2}$ over $(0,2 \pi)$, the random variables

$$
\xi=\int_{0}^{2 \pi} g(u) d z(u), \quad \eta=\int_{0}^{2 \pi} h(u) d z(u)
$$

are well defined, and we have

$$
E(\xi \bar{\eta})=\int_{0}^{2 \pi} \int_{0}^{2 \pi} g(u) \overline{h(v)} f(u, v) d u d v .
$$

The function $F(u, v)$ is called the spectral function of the $x_{n}$ process, and is said to define the spectral distribution of the process, which is a distribution of complex-valued "mass" over the square $C$, such that every surface element $d u d v$ carries the mass $f(u, v) d u d v$. The function $f(u, v)$ is the spectral density of the process, while $z(u)$ defines the corresponding spectral process.

I have elsewhere (Cramér, 2) given a sufficient condition that a harmonizable process will be deterministic. In the present note, $I$ shall be concerned with an $x_{n}$ process as defined by (2), and such that the corresponding spectral density $f(u, v)$ satisfies the conditions $(A)$ and $(B)$. It is proposed to find; for this process, the conditions under which an arbitrarily given sequence of integers $r_{k}$ will constitute the complete set of points of indetermination of the process.

3. Suppose that we are given an $x_{n}$ process as defined by (2), with a spectral function $F(u, v)$ satisfying $(3)$, and a spectral density $f(u, v)$ satisfying the conditions $(A)$ and $(B)$. Let there further be given an increasing sequence of integers:

$$
\cdots<r_{-1}<r_{0}<r_{1}<\cdots,
$$

which may be finite or infinite, in one or both directions.

In order that the given $r_{k}$ sequence will constitute the complete set of points of indetermination of the given $x_{n}$ process, the following condition is necessary and sufficient. The spectral function $F(u, v)$ should admit a development of the form:

$$
F(u, v)=\sum_{r_{k}} \varphi_{k}(u) \overline{\varphi_{k}(v)}+G(u, v),
$$

where $G(u, v)$ is the spectral function of a deterministic harmonizable process, while the series in the second member converges absolutely for all $u$ and $v$, and we have 


$$
\varphi_{k}(u)=\sum_{n=r_{k}}^{\infty} \alpha_{n r_{k}} e^{-i n u}+\beta_{k} u+\gamma_{k}
$$

the one-sided trigonometric series occurring here being absolutely convergent for all $u$, while the $\alpha, \beta$ and $\gamma$ are constants such that $\alpha_{r_{k} r_{k}} \neq 0$ for every $r_{k} \neq 0, \beta_{k}=0$ for $r_{k}>0$, and the series

$$
\sum_{r_{k} \leqq n}\left|\alpha_{n r_{k}}\right|^{2}
$$

extended over all $r_{k} \leqq n$, converges for every fixed $n$.

We shall first show that the condition is necessary. Since by hypothesis $r_{k}$ is a point of indetermination of the given $x_{n}$ process, the innovation

$$
c_{r_{k} r_{k}} \xi_{r_{k}}=x_{r_{k}}-P_{r_{k-1}}\left(x_{r_{k}}\right)
$$

is not identically zero. As in the preceding section, we may take $c_{\tau_{k} r_{k}}$ real and positive, and such that $E\left|\xi_{r_{k}}\right|^{2}=1$. As before we write

observing that we have

$$
c_{n r_{k}}=E\left(x_{n} \bar{\xi}_{r_{k}}\right)
$$

$$
c_{n r_{k}}=0 \text { for } n<r_{k},
$$

as the innovation associated with $\xi_{r_{k}}$ does not enter into the process until the time point $r_{k}$, and is thus uncorrelated with every $x_{n}$ preceding this time point.

We now define a random variable $w$ by writing

$$
w=\int_{0}^{2 \pi} u d z(u)
$$

where $z(u)$ is the spectral process appearing in (2). The stochastic integral is defined in the way indicated in the preceding section. We further write

and

$$
d_{k}=\boldsymbol{E}\left(w \bar{\xi}_{r_{k}}\right)
$$

$$
\varphi_{k}(u)=-\frac{1}{2 \pi i} \sum_{n=r_{k}}^{\infty} \frac{c_{n r_{k}}}{n} e^{-i n u}+\frac{1}{2 \pi} c_{0 r_{k}}(u+\pi)-\frac{1}{2 \pi} d_{k},
$$

where the accent on the summation sign indicates that, if the value $n=0$ falls between the limits of summation, the corresponding term should be omitted. Clearly this is an expression of the form postulated in (7). In order to show that the trigonometric series appearing here is absolutely convergent, we observe that

$$
\left|c_{n r_{k}}\right|^{2} \leqq E\left|x_{n}\right|^{2} E\left|\xi_{r_{k}}\right|^{2}=E\left|x_{n}\right|^{2}=\int_{0}^{2 \pi} \int_{0}^{2 \pi} e^{i n(u-v)} f(u, v) d u d v
$$

Thus by condition $(A)$ the quantities $\left|c_{n r_{k}}\right|^{2}$ are, for a fixed $r_{k}$, the Fourier coefficients of a function in $L^{2}$, so that the series $\sum_{n}\left|c_{n r_{k}}\right|^{4}$ is convergent. Hence by Hölder's inequality it is easily shown that $\sum_{n}^{\prime} \frac{c_{n r_{n}}}{n}$ is absolutely convergent. It has 
H. CRAMÉR, On the linear prediction problem for certain stochastic processes

already been remarked in connection with (1) that the series $\sum_{r_{k} \leq n}\left|c_{n r_{k}}\right|^{2}$ is convergent for every $n$. We finally observe that, in consequence of (8), the lower limit of summation in (10) may be replaced by $-\infty$, since the new terms thus introduced all reduce to zero.

We now consider the expression

$$
G_{K}(u, v)=F(u, v)-\sum_{k=-K}^{K} \varphi_{k}(u) \overline{\varphi_{k}(v)},
$$

with appropriate modification in case the $r_{k}$ sequence is finite in either direction. We shall first show that $G_{K}(u, v)$ is, for every $K$, the covariance function of some random variable $z_{R}(u)$ defined for every $u$ in $(0,2 \pi)$, so that

$$
G_{K}(u, v)=E\left(z_{K}(u) \overline{z_{K}(v)}\right)
$$

for all $u$ and $v$ in $(0,2 \pi)$. In order to show that $G_{K}(u, v)$ is a covariance, it is sufficient to show (cf. Loève, 1 ) that

$$
\int_{0}^{2 \pi} \int_{0}^{2 \pi} q(u) \overline{q(v)} G_{K}(u, v) d u d v \geqq 0
$$

for any continuous $q(u)$. Obviously it is even sufficient to show that (12) holds for any trigonometric polynomial $q(u)$. Taking

$$
\begin{aligned}
& q(u)=\sum_{n=A}^{B} \lambda_{n} e^{i n u}, \\
& Q(u)=\sum_{n=A}^{B} \frac{\lambda_{n}}{i n}\left(e^{i n u}-1\right)+\lambda_{0}(u-2 \pi),
\end{aligned}
$$

we have

By (3) we have

$$
Q^{\prime}(u)=q(u), \quad Q(2 \pi)=0 .
$$

$$
F(u, 0)=F(0, v)=0,
$$

so that we obtain by partial integration, using (4), (5) and (9),

$$
\begin{aligned}
\int_{0}^{2 \pi} \int_{0}^{2 \pi} q(u) \overline{q(v)} F(u, v) d u d v=\int_{0}^{2 \pi} \int_{0}^{2 \pi} Q(u) \overline{Q(v)} f(u, v) d u d v= \\
=E\left|\sum_{A}^{B} \frac{\lambda_{n}}{i n}\left(x_{n}-x_{0}\right)+\lambda_{0}\left(w-2 \pi x_{0}\right)\right|^{2} .
\end{aligned}
$$

On the other hand

$$
\int_{0}^{2 \pi} \int_{0}^{2 \pi} q(u) \overline{q(v)} \varphi_{k}(u) \overline{\varphi_{k}(v)} d u d v=\left|\int_{0}^{2 \pi} q(u) \varphi_{k}(u) d u\right|^{2}
$$

and by some simplè calculation we find

$$
\begin{aligned}
\int_{0}^{2 \pi} q(u) \varphi_{k}(u) d u=-\sum_{n=A}^{B} \frac{\lambda_{n}}{i n}\left(c_{n r_{k}}-c_{0 r_{k}}\right)-\lambda_{0}\left(d_{k}-2 \pi c_{0 r_{k}}\right)= \\
=-E\left\{\left(\sum_{A}^{B} \frac{\lambda_{n}}{i n}\left(x_{n}-x_{\theta}\right)+\lambda_{\theta}\left(w-2 \pi x_{0}\right)\right) \xi_{r_{k}}\right\} .
\end{aligned}
$$


Writing

$$
X=\sum_{A}^{B}, \frac{\lambda_{n}}{i n}\left(x_{n}-x_{0}\right)+\lambda_{0}\left(w-2 \pi x_{0}\right)
$$

we obtain from (11), (13), (14) and (15)

$$
\int_{0}^{2 \pi} \int_{0}^{2 \pi} q(u) \overline{q(v)} G_{K}(u, v) d u d v=E|X|^{2}-\sum_{k}\left|E\left(X \bar{\xi}_{r_{k}}\right)\right|^{2} .
$$

The $\xi_{r_{k}}$ being orthogonal random variables, (12) now follows directly from Bessel's inequality.

Thus $G_{K}(u, v)$ as defined by (11) is always a covariance function. It follows that $G_{K}(u, u) \geqq 0$, so that, allowing $K$ to tend to infinity, the sum over all $k$

$$
\sum_{k}\left|\varphi_{k}(u)\right|^{2}
$$

is convergent, and consequently by the Schwarz inequality

$$
\sum_{k} \varphi_{k}(u) \overline{\varphi_{k}(v)}
$$

is absolutely convergent. Thus $G_{K}(u, v)$ tends to a limit $G(u, v)$ as $K \rightarrow \infty$.

The limit of a sequence of covariance functions being itself a covariance function (cf. Loève 1), we have now proved that $F(u, v)$ admits a development of the form (6), where $G(u, v)$ is a covariance function, while $\varphi_{k}(u)$ has the form (7), the stated convergence conditions being satisfied. It remains to show that $G(u, v)$ is the spectral function of a deterministic harmonizable process.

In order that a covariance function $G(u, v)$ defined in the square $C: 0 \leqq u ; v$ $\leqq 2 \pi$, should be the spectral function of some harmonizable process, it is necessary and sufficient that $G(u, v)$ should be of bounded variation over $C$. We shall first show that this property holds here.

Since $G(u, v)$ is a covariance function, there exists for every $u$ in $(0,2 \pi)$ a random variable $Z(u)$ such that

$$
G(u, v)=F(u, v)-\sum_{k} \varphi_{k}(u) \overline{\varphi_{k}(v)}=E(Z(u) \overline{Z(v)}) .
$$

For an arbitrary sub-interval $(u, u+h)$ of $(0,2 \pi)$ we then have, taking differences in the obvious way,

and thus

$$
\Delta_{2} G(u, u)=\Delta_{2} F(u, u)-\sum_{k}\left|\Delta \varphi_{k}(u)\right|^{2}=E|\Delta Z(u)|^{2},
$$

Let now

$$
E|\Delta Z(u)|^{2} \leqq \Delta_{2} F(u, u)
$$

$$
\begin{aligned}
& 0=u_{0}<u_{1}<\cdots<u_{m}=2 \pi, \\
& 0=v_{0}<v_{1}<\cdots<v_{n}=2 \pi,
\end{aligned}
$$

be two arbitrary sub-divisions of the interval $(0,2 \pi)$. All differences occurring in the sequel will be understood to be related in the obvious way to the sub-intervals in these divisions. 


\section{H. CRAMÉR, On the linear prediction problem for certain stochastic processes}

From (16) we obtain

$$
\Delta_{2} G\left(u_{r}, v_{s}\right)=E\left(\Delta Z\left(u_{r}\right) \overline{\Delta Z\left(v_{s}\right)}\right),
$$

and further by the Schwarz inequality, using (17),

$$
\begin{aligned}
\left|\Delta_{2} G\left(u_{r}, v_{s}\right)\right| & \leqq E\left\{\left|\Delta Z\left(u_{r}\right)\right| \cdot\left|\Delta Z\left(v_{s}\right)\right|\right\} \\
& \leqq \sqrt{E\left|\Delta Z\left(u_{r}\right)\right|^{2} \cdot E\left|\Delta Z\left(v_{s}\right)\right|^{2}} \\
& \leqq \sqrt{\Delta_{2} F\left(u_{r}, u_{r}\right) \cdot \Delta_{2} F\left(v_{s}, v_{s}\right)} .
\end{aligned}
$$

Summing over all the sub-intervals in both variables, we thus obtain by means of the condition $(B)$, assuming that all sub-intervals are sufficiently small,

$$
\left.\sum_{r, s} \mid \Delta_{2} G\left(u_{r}, v_{s}\right)\right) \mid \leqq \sum_{r} \sqrt{\Delta_{2} F\left(u_{r}, u_{r}\right)} \cdot \sum_{s} \sqrt{\Delta_{2} F\left(v_{s}, v_{s}\right)} \leqq 4 \pi^{2} M,
$$

which shows that $G(u, v)$ is of bounded variation over $C$.

Comparing now the general representation (1) of a non-deterministic $x_{n}$ process, and the development (6) of the spectral function $F(u, v)$ in the present case, it is easily verified that the term $\sum \varphi_{k}(u) \overline{\varphi_{k}(v)}$ in (6), where $\varphi_{k}(u)$ is determined by (10), is the spectral function of the sum $\sum c_{n r_{k}} \xi_{r_{k}}$ in (1), while the remaining term $G(u, v)$ is the spectral function of the deterministic component $y_{n}$ of the $x_{n}$ process. We have thus completed the proof that the given condition is necessary.

The proof that the condition is also sufficient is now very simple. We first observe that the predictionary properties (prediction being always understood in the sense of linear least squares prediction) of a stochastic process are entirely determined by the covariance function of the process. Thus if we are given an $x_{n}$ process with the covariance function $R(m, n)$, and if we can show that $R(m, n)$ is the covariance function of some stochastic process having the given sequence of the $r_{k}$ for its points of indetermination, it follows that the given $x_{n}$ process will have precisely the same points of indetermination. For a harmonizable process, the covariance function is uniquely determined by the spectral function $F(u, v)$, so that the same remark applies here to $F(u, v)$.

Suppose now that we are given an $r_{k}$ sequence, and an $x_{n}$ process with a spectral function $F(u, v)$ satisfying all our conditions. In particular, $F(u, v)$ will then be given by the development (6), where $\varphi_{k}(u)$ is given by (7). According to the remark just made, we shall then only have to show that $F(u, v)$ is the spectral function of some stochastic process having the given $r_{k}$ for its points of indetermination.

Consider a stochastic process $x_{n}^{*}$ represented in the form (1), where we take

$$
\begin{array}{ll}
c_{n r_{k}}=-2 n \pi i \alpha_{n r_{k}} \text { for } r_{k} \leqq n, n \neq 0, \\
c_{0 r_{k}}=2 \pi \beta_{k} \quad \text { for } r_{k} \leqq 0,
\end{array}
$$

while the $\xi_{r_{k}}$ are orthogonal random variables, and also orthogonal to the $y_{n}$, which are the variables of a deterministic process with the spectral function $G(u, v)$ appearing in (6). Then it will be immediately seen that the $x_{n}^{*}$ process has the given $r_{k}$ sequence for its points of indetermination, so that the proof is hereby completed. 
We finally remark that the development of the difference $F(u, v)-G(u, v)$, which follows from $(6)$, is formally analogous to the well-known development of the kernel $F(u, v)-G(u, v)$ in terms of its characteristic functions. However, it is easily shown by means of examples that the $\varphi_{k}(u)$ appearing in (6) are not necessarily identical with the characteristic functions of the corresponding kernel.

\section{RE F E R E N CES}

CraMÉr, H., 1. A contribution to the theory of stochastic processes. Proceedings of the Second Berkeley Symposium on Mathematical Statistics and Probability. Berkeley 1950 l.

2. Remarques sur le problème de prédiction pour certaines classes de processus stochastiques. To appear in the Publications de l'Institut de Statistique de l'Lniversité de Paris.

Dоoв, J. L., Stochastic processes. New York (Wiley) 1953.

LoÈve, M., Fonctions aléatoires du second ordre. Supplément to P. LÉYy, Processus stochastiques et mouvement brownien. Paris. (Gauthier-Villars) 1948.

Wrener, N., Extrapolation, interpolation and smoothing of stationary time series. New York (Wiley) 1949.

Wold, H., A study in the analysis of stationary time series. Thesis, Stockholm. Uppsala 1938. 